Ferrata Storti Foundation

\title{
The new small tyrosine kinase inhibitor ARQ531 targets acute myeloid leukemia cells by disrupting multiple tumor-addicted pro- grams
}

Haematologica 2020

Volume 105(10):2420-2431

\section{Correspondence:}

MICHELE CEA

michele.cea@unige.it

Received: April 23, 2019.

Accepted: October 10, 2019.

Pre-published: November 7, 2019.

doi:10.3324/haematol.2019.224956

(C2020 Ferrata Storti Foundation

Material published in Haematologica is covered by copyright. All rights are reserved to the Ferrata Storti Foundation. Use of published material is allowed under the following terms and conditions:

https://creativecommons.org/licenses/by-nc/4.0/legalcode. Copies of published material are allowed for personal or internal use. Sharing published material for non-commercial purposes is subject to the following conditions:

https://creativecommons.org/licenses/by-nc/4.0/leǵalcode, sect. 3. Reproducing and sharing published material for commercial purposes is not allowed without permission in writing from the publisher.
Debora Soncini, ${ }^{1}$ Stefania Orecchioni, ${ }^{2}$ Samantha Ruberti, ${ }^{1}$ Paola Minetto, ${ }^{1,3}$ Claudia Martinuzzi, ${ }^{1}$ Luca Agnelli, ${ }^{4}$ Katia Todoerti, ${ }^{4}$ Antonia Cagnetta, ${ }^{1,3}$ Maurizio Miglino, ${ }^{1,3}$ Marino Clavio, ${ }^{1,3}$ Paola Contini, ${ }^{5}$ Riccardo Varaldo, ${ }^{6}$ Micaela Bergamaschi, ${ }^{1}$ Fabio Guolo, ${ }^{1}$ Mario Passalacqua, ${ }^{7}$ Alessio Nencioni, ${ }^{5}$ Fiammetta Monacelli, ${ }^{5}$ Marco Gobbi, ${ }^{1,3}$ Antonino Neri, ${ }^{4}$ Giovanni Abbadessa, ${ }^{8}$ Sudharshan Eathiraj, ${ }^{8}$ Brian Schwartz, ${ }^{8}$ Francesco Bertolini, ${ }^{2}$ Roberto M. Lemoli ${ }^{1,3}$ and Michele Cea ${ }^{1,3}$

${ }^{1}$ Chair of Hematology, Department of Internal Medicine and Specialities (DiMI), University of Genoa, Genoa, Italy; '2Laboratory of Hematology-Oncology, European Institute of Oncology IRCCS, Milan, Italy; ${ }^{3}$ IRCCS Ospedale Policlinico San Martino, Genoa, Italy; ${ }^{4}$ Department of Oncology and Hemato-Oncology, University of Milan, Milan, Italy; ${ }^{5}$ Department of Internal Medicine and Specialities (DiMI), University of Genoa, Genoa, Italy; ${ }^{6}$ Division of Hematology and Hematopoietic Stem Cell Transplantation Unit, Ospedale Policlinico San Martino, Genoa, Italy; ${ }^{7}$ Department of Experimental Medicine (DIMES), University of Genoa, Genoa, Italy and ${ }^{8}$ ArQule, Burlington, MA, USA

\section{ABSTRACT}

yrosine kinases have been implicated in promoting tumorigenesis of several human cancers. Exploiting these vulnerabilities has been shown to be an effective anti-tumor strategy as demonstrated for example by the Bruton tyrosine kinase (BTK) inhibitor, ibrutinib, for treatment of various blood cancers. Here we characterize a new multiple kinase inhibitor, ARO531, and evaluate its mechanism of action in preclinical models of acute myeloid leukemia. Treatment with ARO531, by producing global signaling pathway deregulation, resulted in impaired cell cycle progression and survival in a large panel of leukemia cell lines and patient-derived tumor cells, regardless of the specific genetic background and/or the presence of bone marrow stromal cells. RNA-sequencing analysis revealed that ARO531 constrained tumor cell proliferation and survival through BTK and transcriptional program dysregulation, with proteasome-mediated MYB degradation and depletion of shortlived proteins that are crucial for tumor growth and survival, including ERK, MYC and MCL1. Finally, ARO531 treatment was effective in a patient-derived leukemia mouse model, causing significant impairment of tumor progression and survival, at tolerated doses. These data justify the clinical development of ARO531 as a promising targeted agent for the treatment of patients with acute myeloid leukemia.

\section{Introduction}

Acute myeloid leukemia (AML) is an aggressive disease characterized by uncontrolled clonal proliferation of abnormal myeloid progenitor cells in the bone marrow and blood. Despite recent advances in its treatment, as many as $70 \%$ of patients aged 65 or older will die within 1 year of diagnosis. The efficacy of standard high-dose chemotherapy and stem cell transplantation is limited by treatment-related morbidity and mortality, especially in elderly patients. ${ }^{1.3}$ Cancer treatment is undergoing a significant revolution from "one-size-fits-all" cytotoxic therapies to tailored approaches that target molecular alterations precisely. Notably, precision medicine, by linking specific genetic anomalies of tumors with available targeted therapies, is emerging as an innovative approach for AML treatment, with development of breakthrough drugs targeting specific molecular features (e.g., 
FLT3 and IDH1/2 inhibitors). ${ }^{46}$ However, identification of patients who will benefit from targeted therapies is more complex than simply identifying patients whose tumors harbor the targeted aberration. A rational combination of therapeutic agents may prevent the development of resistance to therapy, with molecular strategies aimed at targeting multiple pathways resulting in a more effective treatment across cancer subtypes.

The Bruton tyrosine kinase (BTK), a member of the TEC family kinases, is a critical terminal kinase enzyme in the B-cell antigen receptor signaling pathway. ${ }^{7,8}$ Its activation leads to BTK phosphorylation which in turn results in downstream events such as proliferation, immune function alteration and survival through multiple signaling cascades. ${ }^{9}$ Chronic activation of BTK-mediated signaling represents a key driver for a number of types of cancers, ${ }^{10-14}$ including AML. ${ }^{15-22}$ Therefore, new inhibitors are needed to target tyrosine kinases better in these patients. Recent studies have shown that oncogenic cellular dysregulation is critical for the activity of the anti-BTK targeting agent ibrutinib, ${ }^{23,24}$ and that co-treatment with BET protein bromodomain antagonists or BCL-2 inhibitors may enhance the efficacy of ibrutinib in tumor cells..$^{25,26}$

Herein we characterize ARO531, a reversible small molecule inhibitor of BTK and several additional kinases, in preclinical models of AML. We provide evidence that ARQ531 greatly compromises survival of AML cells by inducing a "one shot" inhibition of multiple oncogenic transcriptional pathways. This resulted in potent antiAML activity in a patient-derived xenograft AML mouse model, providing the rationale for future clinical trials.

\section{Methods}

\section{Reagents}

ARO531 was provided by ArQule, Inc (Burlington, MA, USA). The compound was dissolved in dimethylsulfoxide (Sigma-Aldrich) and stored at $10 \mathrm{mM}$ at $-80^{\circ} \mathrm{C}$ for experiments. Ibrutinib, daunorubicin, cytarabine and MG132 were purchased from Selleck Chemicals LLC (Houston, TX, USA). ZVAD-FMK was purchased from Promega (catalog n. G7232).

\section{Patient-derived xenograft acute myeloid leukemia cells}

Experiments were carried out on 6- to 8-week old, nonobese diabetic severe combined immunodeficient (NOD/SCID) interleukin-2 receptor $\gamma$ (IL-2Rg)-null (NSG) mice. The NSG mice were bred and housed under pathogen-free conditions in the animal facilities at the European Institute of Oncology-Italian Foundation for Cancer Research Institute of Molecular Oncology (IEOIFOM, Milan, Italy). All animal experiments were carried out in strict accordance with Italian laws (Legislative Decree 26/2014 and subsequent amendments) and were approved by the institutional committee. NSG mice were engrafted with 300,000 primary human AML cells (M4, acute myelomonocytic leukemia with wild-type FLT3). On day 19 after introduction of the AML cells, once a systemic xenograft had been confirmed, mice were randomized into three groups: vehicle-treatment group ( $n=5)$, low-dose ARO531 treatment group ( $25 \mathrm{mg} / \mathrm{kg} ; \mathrm{n}=5)$ and high-dose ARO531 treatment group ( $37.5 \mathrm{mg} / \mathrm{kg} ; \mathrm{n}=5$ ) and the percentage of human leukemic cells in peripheral blood was measured weekly until day 42 . The phenotype of human cells in NSG mice was evaluated using the following anti-human antibodies: anti-CD117-PeCy7 (IMMU 103.44), anti-CD45-APC (J.33), anti-CD34-APCCy7 (D3HL60.251) from Beckman-Coulter (Irving, TX, USA) and anti-mouse CD45-PE (30-F11) from BD Biosciences to exclude murine cell contamination. Cell suspensions were evaluated by a three-laser, ten-color flow cytometer (Navios, Beckman Coulter, Brea, CA, USA) using analysis gates designed to exclude dead cells, platelets, and debris. Percentages of stained cells were determined and compared to appropriate negative controls. Seven-aminoactinomycin D (7AAD) from SigmaAldrich was used to enumerate viable, apoptotic, and dead cells.

\section{Statistical analyses}

All in vitro experiments were repeated at least three times and performed in triplicate; a representative experiment is shown in each figure. All data are shown as mean \pm standard deviation (SD). The Student $t$ test was applied to compare two experimental groups using Graph-Pad Prism software (http://www.graphpad.com). The minimal level of significance was specified as $P<0.05$. Survival analysis was performed by the Kaplan-Meier method, and the log-rank test was used to compare survival differences. Drug interactions were assessed by CalcuSyn 2 software (Biosoft), which is based on the Chou-Talalay method. A combination index (CI) of 1 indicates an additive effect; a $\mathrm{CI}<1$ indicates synergism and a $\mathrm{CI}>1$ indicates antagonism.

\section{Results}

\section{ARQ531 shows strong anti-acute myeloid leukemia activity but preserves normal hematopoietic stem cells}

In line with previously reported data, ${ }^{15,16}$ we observed that BTK is frequently dysregulated in AML, with mRNA levels being significantly higher than in other cancer types (Online Supplementary Figure S1). To confirm its relative abundance, we screened a representative panel of human AML cell lines and primary blasts for BTK expression and activity by western blot (Figure 1A). Protein was detectable in all AML-screened cells (15/15) and, more importantly, independent of specific mutational profiling. Similarly, BTK activity (measured by Y223 phosphorylation) was observed in FLT3 wild-type and FLT3 mutated cells as well. An analogous investigation was applied to a larger cohort of AML patients derived from The Cancer Genome Atlas database, which showed uniform expression of BTK transcript in different AML subtypes. Overall, these data, by confirming the presence of BTK in AML, support targeting this kinase in this hematologic malignancy, as previously reported..$^{14,15}$

ARQ531 is a recently described, reversible BTK inhibitor with promising activity in mouse models of chronic lymphocytic leukemia and lymphomas. ${ }^{27}$ Based on constitutively active BTK levels observed in AML cells, we evaluated the therapeutic activity of ARQ531 on these cells, using ibrutinib as a control. In vitro efficacy screening was performed on cultured $(n=8)$ and primary $(n=13)$ AML cells, comparing the efficacy of both drugs. As shown in Figure 1B, exposure to ARO531 reduced in vitro viability more than ibrutinib did (Figure 1C). Analysis of the half maximal inhibitory concentration $\left(\mathrm{IC}_{50}\right)$ at $48 \mathrm{~h}$ 
after treatment showed that the cells were more sensitive to ARQ531 than to ibrutinib, which exhibited 10-fold lower activity. (Figure 1D) A significant anti-AML effect of ARO531 was also observed on blasts from AML patients $(n=13)$ regardless of mutational status, European LeukemiaNet risk, and surface expression of CD117 (Figure 1E, Table 1). Consistent with these data, a dosedependent increase in the percentage of apoptotic and dead cells measured by annexin $\mathrm{V}$ and propidium iodide staining was also observed after ARO531 treatment, together with several apoptotic features including caspase 3 and poly(AD-ribose) polymerase cleavage as well as reduction of anti-apoptotic MCL-1 and BCL-2 protein expression (Figure 1F, G and Online Supplementary Figure $S 2 A)$. Viability was completely restored by pre-incubation with the pan-caspase inhibitor Z-VAD (Online Supplementary Figure S2B) In contrast, ibrutinib treatment resulted in weaker effects on apoptosis, thus suggesting that ARO531 is more effective than ibrutinib, probably because it induces downregulation of additional survival mechanisms.

It is well known that the bone marrow microenvironment has a role in the promotion of tumor growth, survival and drug-resistance. ${ }^{28}$ We therefore treated AML cells in the presence of normal or leukemic mesenchymal stromal cells. As expected, normal and AML stroma both protected tumor cells from spontaneous apoptosis; however, the efficacy of ARO531 was preserved, with no significant effect on the viability of mesenchymal stromal cells (data not shown). Indeed, compared to spontaneous apoptosis of blast cells, ARO531 increased cell death in AML cells cultured alone, and preserved its activity in the presence of normal or AML mesenchymal stromal cells, suggesting that ARO531 abrogates the survival benefit from stromal cells (Figure 2A). Overall, our data indicate that ARO531 is a potent anti-AML drug even in the presence of a tumorsupportive microenvironment, and irrespective of FLT3 mutational status. ${ }^{21}$ Finally, ARO531 activity on normal cells was also investigated by employing clonogenic and viability assays in order to measure the impact of treatment on both CD34+ cells and mononuclear cells isolated from the bone marrow and peripheral blood of healthy donors. As shown in Figure 2B-D, all of these cells were largely unaffected by exposure to ARO531 at dose levels toxic to tumor cells, proving that ARO531 targets cancer cells without off-target effects on hematopoietic stem cells, resulting in a favorable therapeutic index.

\section{BTK signaling inhibition partially contributes to the anti-leukemic activity of ARQ531}

Based on its reported activity, we first studied the effect of ARQ531 on BTK signaling by analyzing tumor cell migration. ${ }^{27,29 \cdot 31} \mathrm{~A}$ transwell assay system was employed to investigate the role of the stromal cell-derived factor 1 (SDF-1)/CXCR4 axis in the anti-AML activity of ARO531. As shown in Online Supplementary Figure S3, ARO531 reduced tumor cell migration in response to SDF-1 by $66 \%(P=0.001)$, suggesting similar activity to ibrutinib (71\% reduction; $P<0.001)$.

Next, to confirm the role of BTK in the anti-AML activity of ARO531, we investigated its effect on BTK signaling in AML cells over a range of concentrations. As shown in Figure 2E, ARO531 treatment completely abrogated the activity of BTK, as measured by Y223 phosphorylation, similar to the effects seen with ibrutinib treatment.
However, as shown in Figure 1B, ARO531 has anti-AML activity even on cells expressing low levels of BTK, suggesting that BTK targeting might not be critical for the activity of ARQ531. To corroborate this hypothesis, we treated BTK-silenced (BTK knocked down, BTK-KD) AML cells with increasing doses of ARO531. ARO531 treatment reduced the viability of both BTK-KD and BTK wild-type cells to about $50 \%$ of control, demonstrating the importance of alternative targets for ARO531 activity in AML (Figure 2F).

\section{ARQ531 treatment suppresses transcriptional oncogenic activity in acute myeloid leukemia cells}

To identify ARO531-induced global perturbations in transcriptional profiling, we generated RNA-sequencing data and performed functional annotation analysis of drug- versus dimethylsulfoxide-treated AML cells. As shown in Online Supplementary Figure S4A, principal component analysis segregated samples based on treatment, suggesting a coherent transcriptional result rather than global, non-specific transcriptional silencing in response to this drug. Indeed, analysis of differential expression identified 377 and 852 genes that were significantly upregulated and downregulated, respectively, with a ratio greater than 2-fold and $P<0.05$. (Figure 3A, B) As a measure of the specificity of this effect, gene set enrichment analysis was performed on the entire set of signatures available from the Molecular Signatures Database (MSigDB). Biological modules associated with oncogenic transcriptional programs (e.g., ribosomal biogenesis and assembly, unfolded protein response stress and MYC) were significantly enriched in ARQ531-suppressed genes (Figure 3C and Online Supplementary Figure S4B). In line with these findings, although treatment did not exert significant suppression of gene sets for factors linked to the pathophysiology of AML, such as C/EBP $\alpha-\beta$, RUNX1, PU.1, ERG and FLI1, a significant reverse correlation was observed for transcriptional signatures of MYC-upregulated target genes, which in turn reflects the selective suppression of its transcriptional networks. (Figure 3D) Indeed, reverse transcriptase polymerase chain reaction analysis of MYC and its target, CDC2, showed consistent downregulation following short-term exposure to the drug (Online Supplementary Figure S4C), pointing to ARO531 as a selective suppressor of the MYC-regulated transcriptional pathway. To further support these data, we used the ARO531 expression signature to query the Library of Integrated Network-Based Cellular Signatures (LINCS) Program ( $w$ ww. lincscloud.org, web interface available at http://amp.pharm.mssm.edu/L1000CDS2/\#/index). As shown in Figure 3E, the most significant ARQ531-correlated signatures were those of oncogenic transcription factor inhibitors (such as fluvastatin, gefitinib and histone deacetylase inhibitors) as well as those related to knockdown of ribosome subunits and translation initiation factors. Together, these data indicate that ARO531 inhibits oncogenic transcriptional pathways in AML cells.

\section{ARQ531 interferes with the pro-survival MAPK pathway in OCI-AML3 cells}

As already reported, ARQ531 is a potent, ATP-competitive, reversible inhibitor of BTK and several additional kinases important to the viability, proliferation, activation, and motility of tumor cells. ${ }^{27}$ Among the most intriguing additional targets of ARO531 are RAF1 and 
A

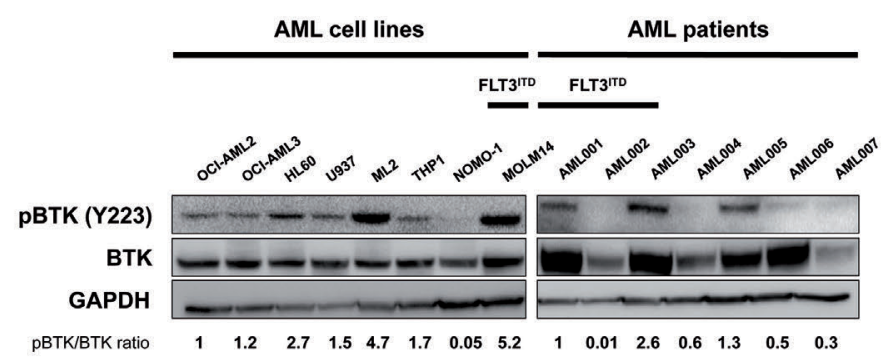

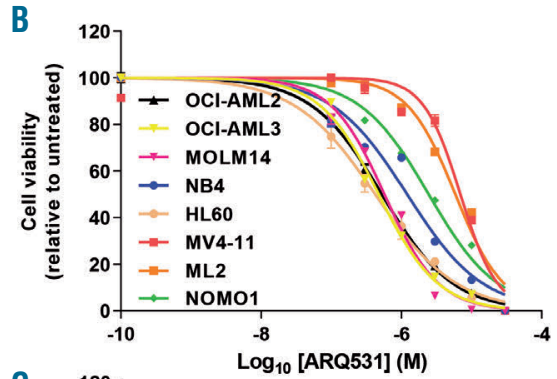

C

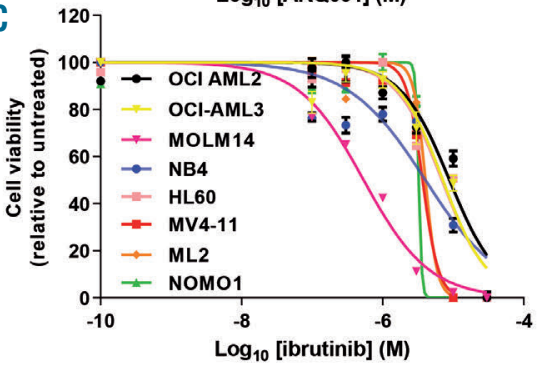

D

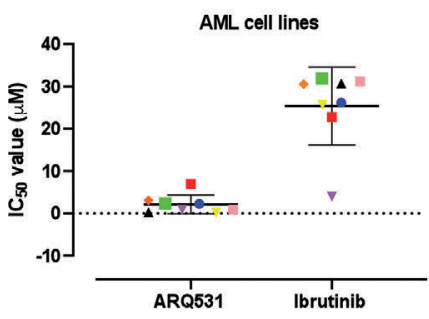

E

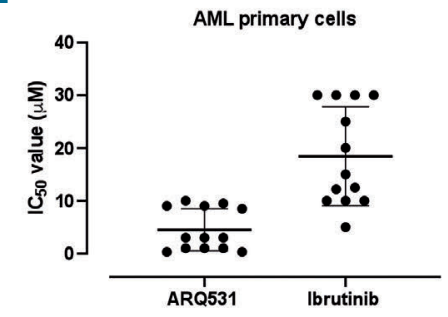

$\mathbf{F}$
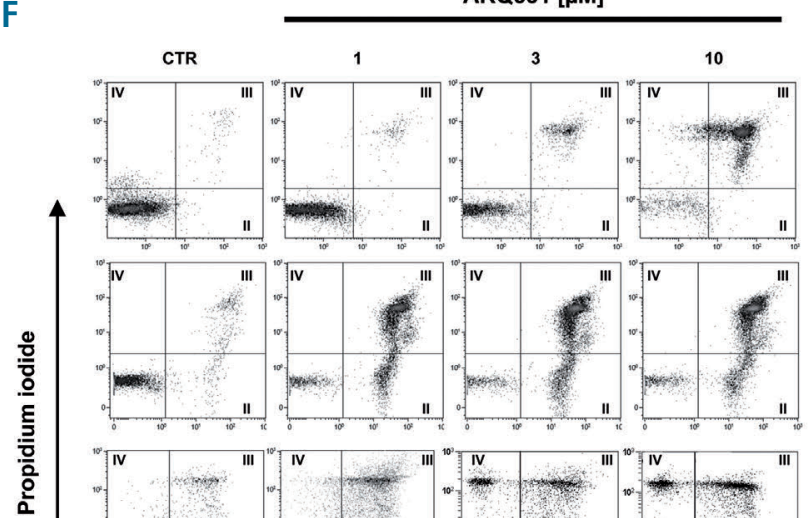

L

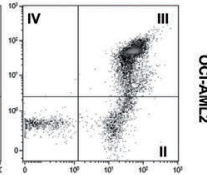

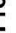
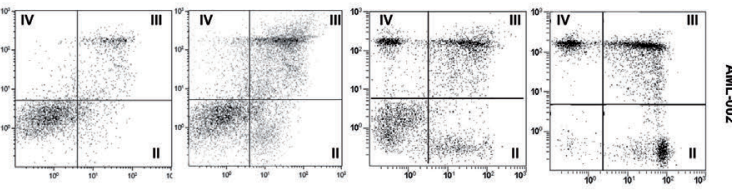

Annexin V
G

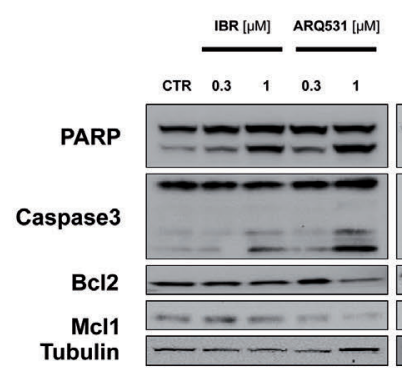

OCI AML3

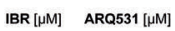

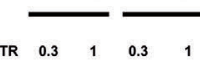

$\begin{array}{lllll}\text { CTR } & 0.3 & 1 & 0.3 & 1\end{array}$

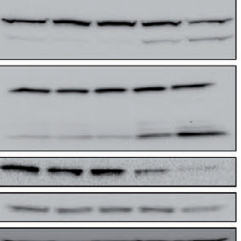

AML-001

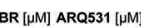

CTR 11

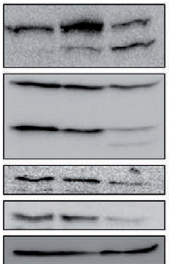

I. Live cells

II. Early apoptosis

III. Late apoptosis

IV. Necrosis

Figure 1. ARQ531 shows strong anti-tumo activity by inducing apoptosis of acute myeloid leukemia cells. (A) Immunoblot for phosphoBTK, BTK and GAPDH (loading control) in the indicated human acute myeloid leukemia (AML) cell lines and primary AML samples regardless of the specific genomic landscape. (B, C) Viability of AML cell lines after treatment with ARQ531 (B) or ibrutinib (C), as measured by MTS assay. The mean + standard deviation (SD) from at least three independent experiments is shown. (D) Half maximal inhibitory concentration $\left(\mathrm{IC}_{50}\right)$ values, measured for each tested cell line as in $(B)$ and $(C)$. (E) Drug effects on primary $A M L$ patient-derived samples $(n=13)$ treated with increasing doses of ARQ531 or ibrutinib (0-30 $\mu \mathrm{M}$ for $48 \mathrm{~h}$ ). $\mathrm{IC}_{50}$ values are visualized for each tested primary AML cell line. (F) HL60, OCl-AML2 and primary AML-002 cells were treated with ARQ531 or dimethylsulfoxide (CTR) in a dosedependent manner for $48 \mathrm{~h}$. Apoptotic cells were detected by annexin $\mathrm{V} /$ propidium iodide staining. Representative dot plots are shown. (G) Immunoblots for PARP, caspase 3, MCL-1, BCL-2 and tubulin on indicated AML cell lines and primary blast cells following treatment with a BTK inhibitor (ARQ531 versus ibrutinib) at $24 \mathrm{~h}$. 
MEK1, constituents of the ERK signaling pathway that is frequently dysregulated in tumor cells. ${ }^{32.34}$ To confirm this activity in AML, cells were treated with increasing doses of ARQ531. As expected, activation of AKT and ERK was inhibited in a dose-dependent manner, likely due to predicted inhibition of RAF1 and MEK1 (Figure 4A). Subsequent experiments confirmed this hypothesis by revealing specific impairment of these kinases after ARQ531 treatment (Figure 4B). To support the pivotal role played by ERK, we exposed cells to the mitogenic effects of a higher serum concentration $(20 \%)$. As shown in Online Supplementary Figure S5A, B, this strategy resulted in enhanced phosphorylation of ERK, which rescued the anti-AML activity of the drug, thus providing evidence of the relevance of ERK in the observed anti-tumor effect. Moreover, consistent with RNA-sequencing analysis, drug exposure resulted in prominent and specific downregulation of the oncogenic transcription factor MYC at the protein level (Figure 4B). Since the MAPK pathway enhances MYC protein stability by inducing its phosphorylation at serine $62,{ }^{35}$ we assessed p-MYC S62
Table 1. Characteristics of the patients with acute myeloid leukemia.

\begin{tabular}{lccccc} 
Patient & $\begin{array}{c}\text { FAB } \\
\text { classification }\end{array}$ & Karyotype & NPIM & FLT3-IID & $\begin{array}{c}\text { ELN risk } \\
\text { group }\end{array}$ \\
AML-013 & AML M2 & normal & mut & wt & low \\
AML-012 & AML M1 & normal & mut & mut & int. \\
\hline AML-011 & AML M6 & complex & wt & wt & high \\
AML-009 & AML M2 & $50 X X$ & wt & wt & high \\
\hline AML-007 & AML M3 & t(15;17) & $/$ & wt & M3 \\
AML-006 & AML M4 & normal & mut & mut & low \\
\hline AML-005 & AML M2 & normal & mut & mut & low \\
AML-004 & AML M4 & normal & mut & wt & low \\
\hline AML-003 & AML M2 & normal & wt & wt & int \\
AML-001 & AML M3 & t(15;17) & $/$ & mut & M3 \\
\hline AML-008 & AML M3 & t(15;17) & $/$ & wt & M3 \\
AML-010 & AML M4 & n.a & wt & wt & int. \\
\hline AML-002 & AML M2 & normal & mut & wt & high \\
\hline
\end{tabular}

FAB: French-American-British; ITD: internal tandem duplication; ELN: European LeukemiaNet; AML: acute myeloid leukemia; mut: mutated; wt: wild-type; int: intermediate; n.a.; not available
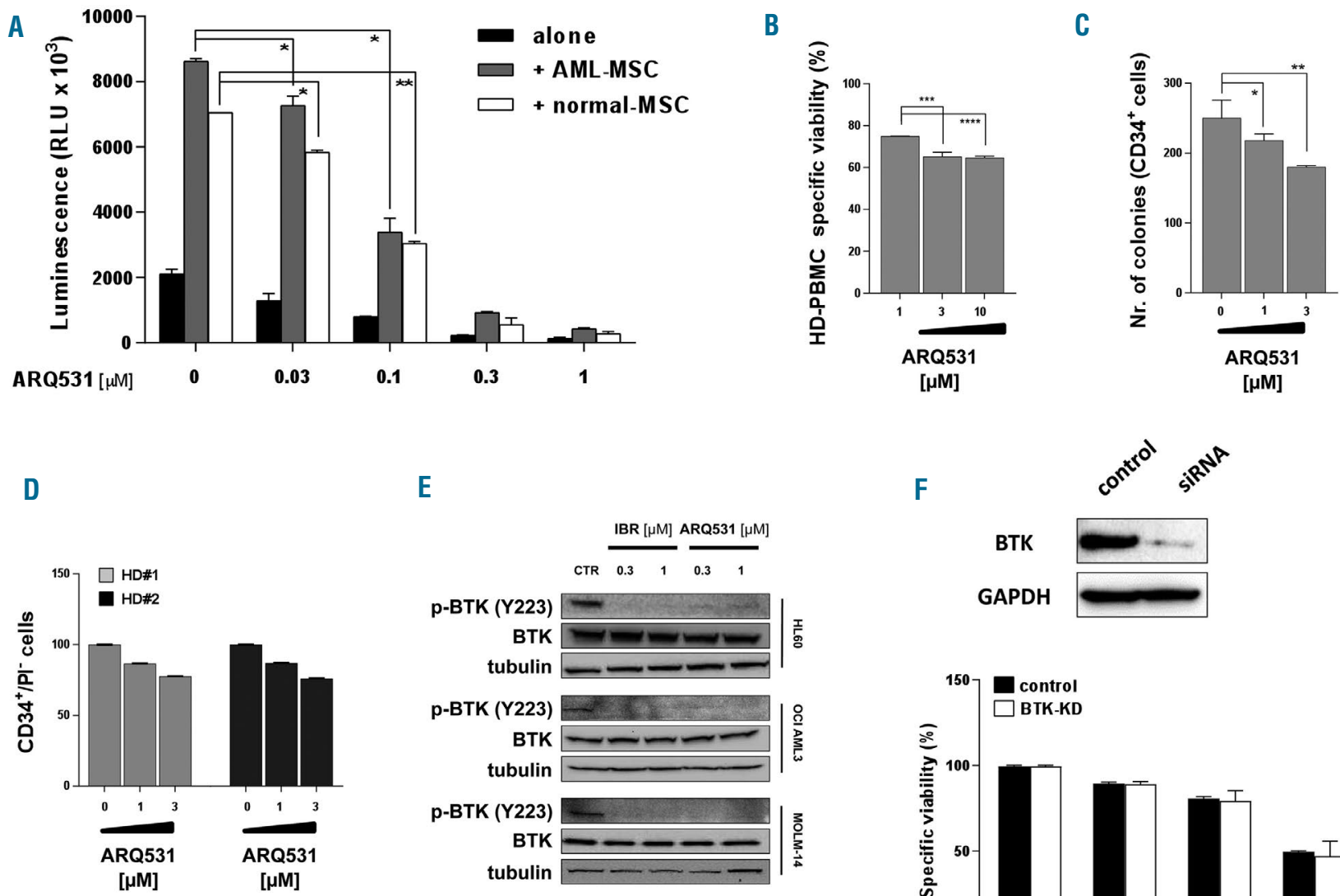

E
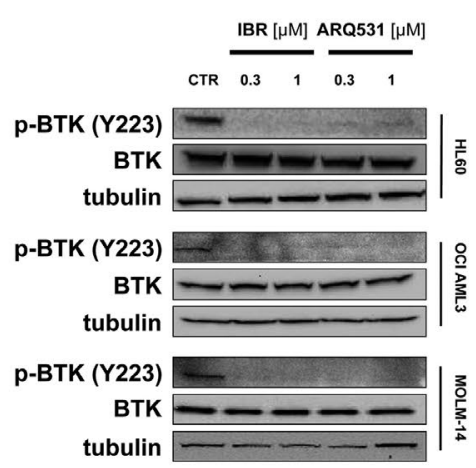

$\mathbf{F}$

BTK

GAPDH
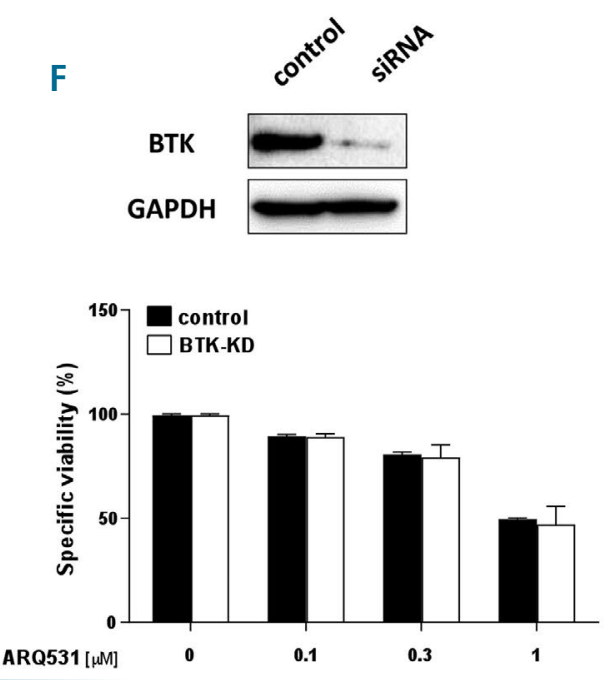

Figure 2. ARQ531 triggers anti-acute myeloid leukemia toxicity regardless of BTK activity and presence of stromal cells but preserves normal hematopoietic stem cells. (A) Viability of OCI-AML2 GFP/luc cells treated with ARQ531 for $48 \mathrm{~h}$, alone and in the presence of normal mesenchymal stem cells (MSC) (blank) or acute myeloid leukemia (AML)-MSC (gray) stroma, measured by a luciferase-based luminescence assay. Data are represented as mean \pm standard deviation (SD) in all histograms $(n=3) .0 .02<* P<0.03 ; * * P<0.05$. (B-D) Healthy donor (HD)-derived hematopoietic precursor (BM-CD34 ${ }^{+}$) and peripheral blood mononuclear cells $(P B M C)$ were exposed to increasing doses of ARQ531, and clonogenic abilities (C) or viability (B, D) were calculated. Colony formation of ARQ531-treated cells (CFC) was measured after 2 weeks. Viability was calculated as propidium iodide (PI)-negative cells among the CD34+ population. Data are represented as mean \pm SD $(n=3)$; unpaired $t$ test, $* * * P<0.001, * * * * P<0.0001$. (E) Western blot showing that ARQ531 treatment effectively abrogates the BTK signaling cascade in three different human AML cell lines (HL60, OCl-AML3 and MOLM14) following $24 \mathrm{~h}$ of treatment. The effect of ibrutinib is also shown as a positive control. (F) Viability of BTK-silenced (nucleofected with specific siRNA targeting BTK) or control HL60 cells (siRNA scramble) treated with increasing doses of ARQ531 for $48 \mathrm{~h}$. Data are data represented as mean $\pm S D$ in all $(n=3)$. 
A

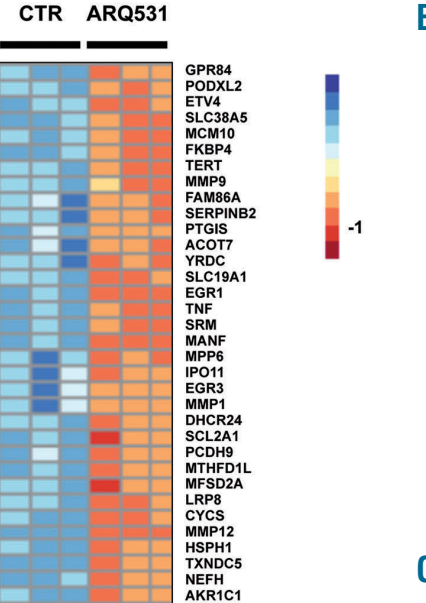

B

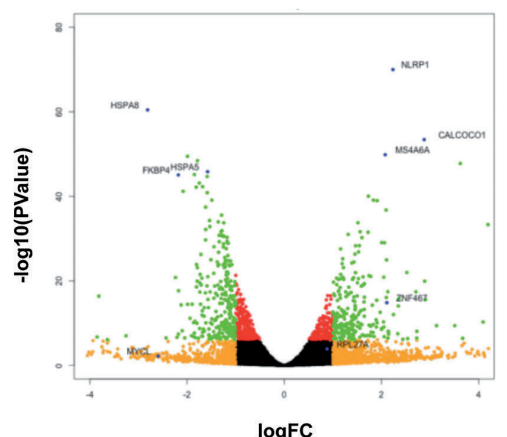

Top gene signatures

\begin{tabular}{|lcccc|}
\hline Gene Set & Size & ES & NES & FDR q-val \\
\hline HALLMARK_MYC_TARGETS_V1 & 87 & $-0,48$ & $-3,01$ & $<0.0001$ \\
HALLMARK_MYC_TARGETS_V2 & 33 & $-0,61$ & $-2,8$ & $<0.0001$ \\
HALLMARK_MTORC1_SIGNALING & 60 & $-0,49$ & $-2,78$ & $<0.0001$ \\
HALLMARK_UNFOLDED_PROTEIN_RESPO & & & & \\
NSE & 26 & $-0,58$ & $-2,54$ & $<0.0001$ \\
MYC_UP.V1_UP & 32 & $-0,61$ & $-2,91$ & $<0.0001$ \\
IL15_UP.V1_UP & 23 & $-0,67$ & $-2,85$ & $<0.0001$ \\
KRAS.600.LUNG.BREAST_UP.V1_UP & 12 & $-0,84$ & $-2,81$ & $<0.0001$ \\
IL2_UP.V1_UP & 15 & $-0,76$ & $-2,73$ & $<0.0001$ \\
MTOR_UP.N4.V1_UP & 20 & $-0,66$ & $-2,64$ & $<0.0001$ \\
RPS14 DN.V1 DN & 27 & $-0,6$ & $-2,64$ & $<0.0001$ \\
\hline
\end{tabular}

D
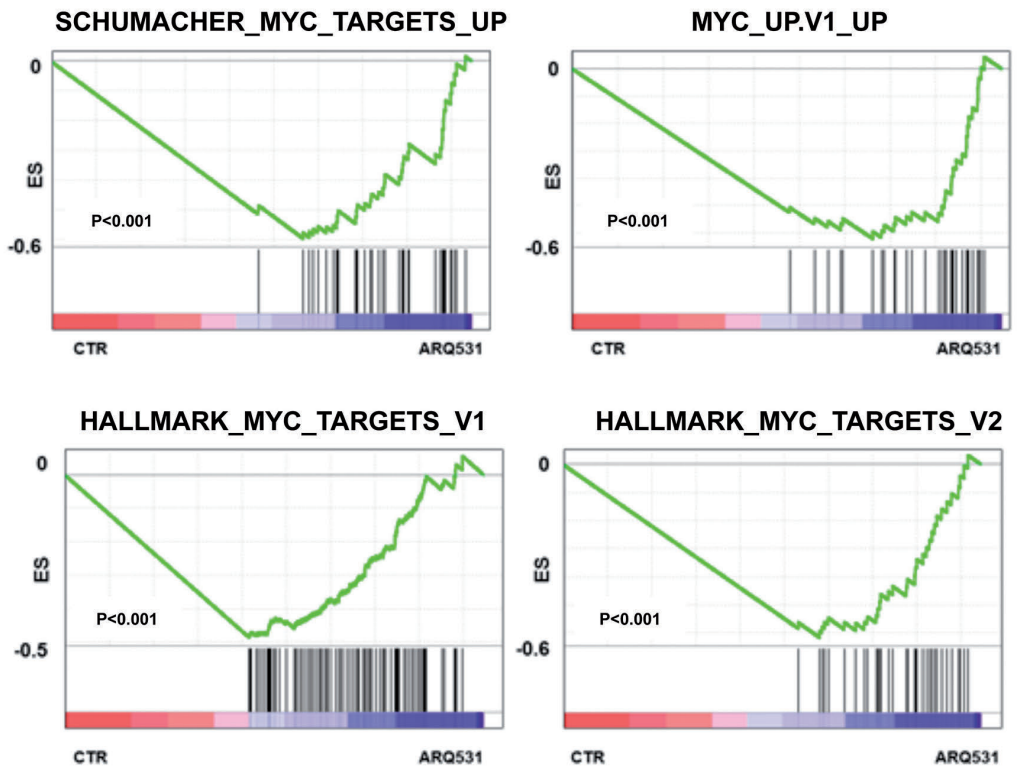

E

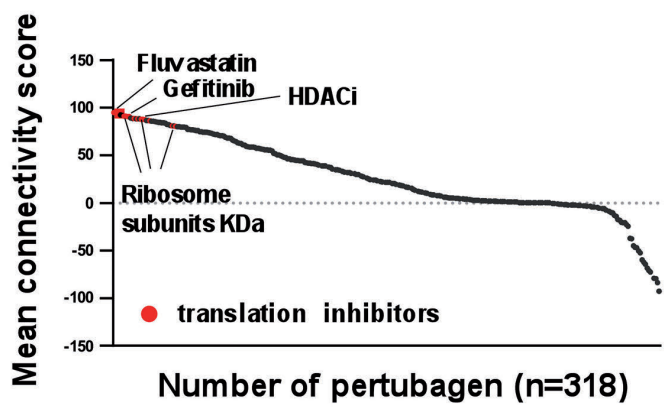

Figure 3. Molecular perturbation triggered by ARQ531 in acute myeloid leukemia cells. (A) Heatmap for the highest 50 down- and up-regulated genes $(P<0.0001)$ following ARQ531 treatment of OCI-AML3 cells. (B) Volcano plot of RNA-sequencing of drug- versus dimethylsulfoxide-treated cells in OCI-AML3 showing that 377 and 852 genes were significantly up- and down-regulated, respectively, with a fold change (FC) $>2$. (C) Table of the ten most significantly enriched gene sets, from the Hallmark collection, enriched with genes downregulated by AR0531 in acute myeloid leukemia (AML) cells. Number of genes in each set (n), the normalized enrichment score (NES), and the test of statistical significance of the false discovery rate (FDR) $q$ value are highlighted. (D) Enrichment plots of the top four most significantly enriched gene sets (MYC-related) in transcriptional profiles of AML cells treated (right) or untreated (left) with ARQ531. (E) Connectivity score generated by the LINCS L1000 Characteristic Direction Signature Search Engine tool, which compared the ARQ531-derived transcriptional profile against 10,000 "perturbagen" signatures (corresponding to shorthairpin RNA, open reading frame and compounds). Top-ranked scores of relevant results are indicated by arrows. HDACi: histone deacetylase inhibitor. 
A

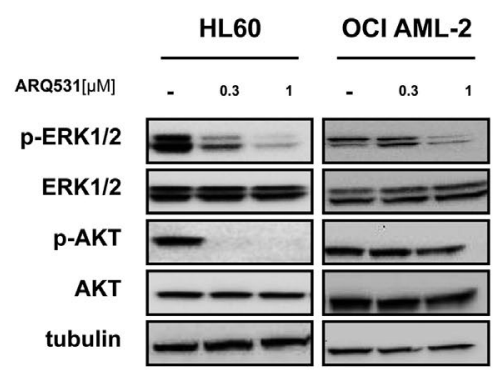

B

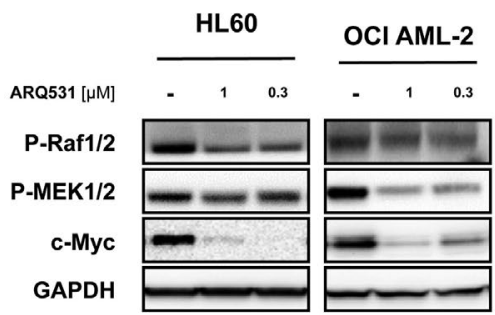

C

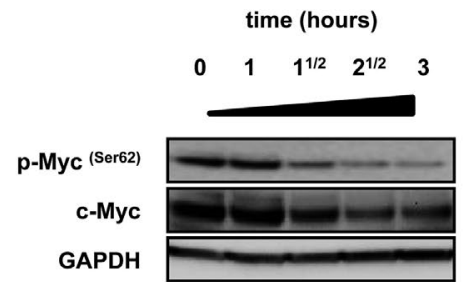

D

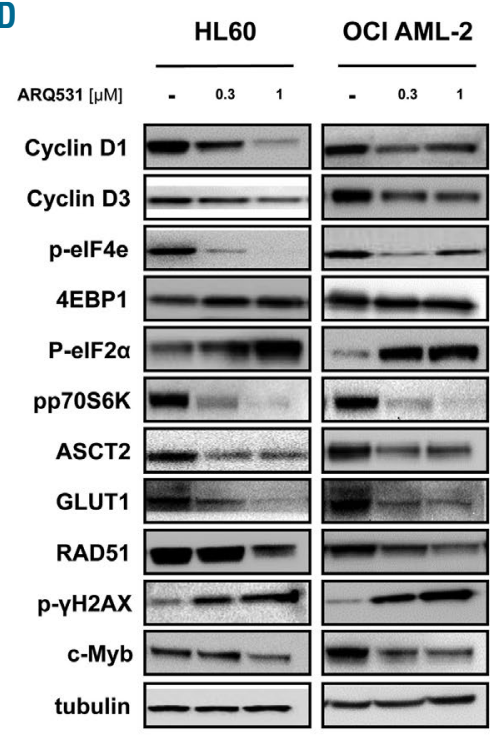

G

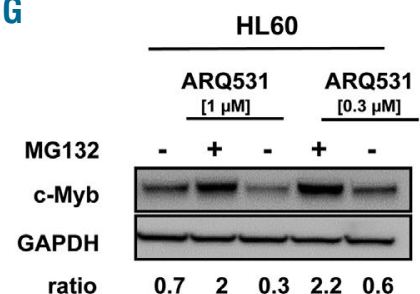

E
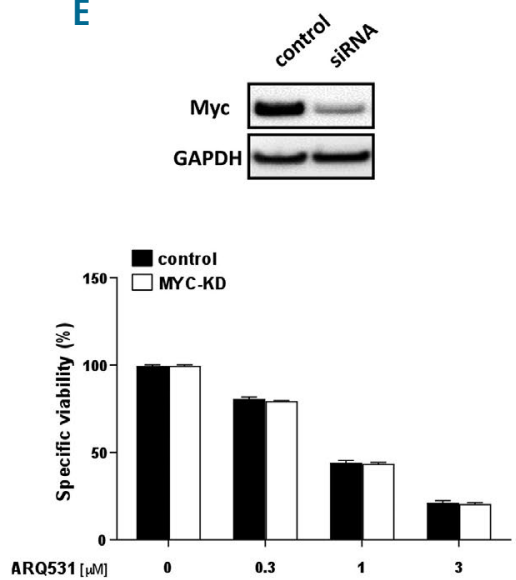

$\mathbf{F}$
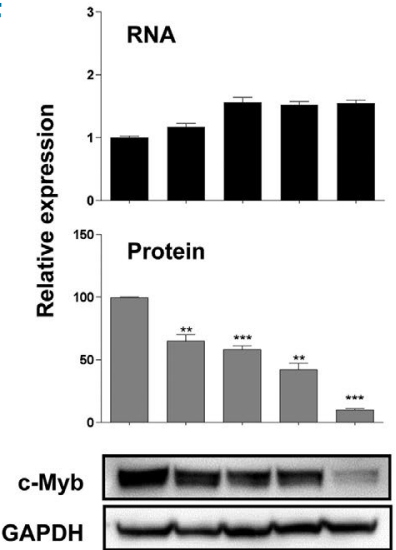

$\begin{array}{lllll}0 & 12 & 18 & 20 & 24\end{array}$

Figure 4. BTK inhibition and MYC/MYB degradation represent molecular bases for the anti-leukemic activity of ARQ531. (A) Western blot showing that $24 \mathrm{~h}$ of treatment with ARQ531 (0.3-1 $\mu \mathrm{M})$ abrogates ERK and AKT activation in HL60 and OCI-AML2 cells. (B) Western blot analysis shows that $24 \mathrm{~h}$ of ARQ531 treatment (0.3-1 $\mu \mathrm{M})$ affects kinases in the RAF/MEK/ERK pathway of acute myeloid leukemia (AML) cell lines, resulting in MYC downregulation. (C) Western blot showing time-dependent effects of ARQ531 exposure on p-MYC S62 and total MYC in the HL60 cell line. (D) Western blot showing deregulation of C-MYCcontrolled signals in AML cells following treatment with ARQ531 at the indicated doses after $24 \mathrm{~h}$. (E) Viability of MYC-silenced or control HL60 cells treated with increasing doses of ARQ531 for $48 \mathrm{~h}$. The mean \pm standard deviation (SD) are shown $(n=3)$. ( $F$ ) Protein and mRNA expression in AML cells after 24 $\mathrm{h}$ treatment with dimethylsulfoxide (DMSO) or the indicated ARQ531 concentrations, normalized to DMSO controls. Bars and error bars are means and SD of three independent experiments. $* P<0.05 ; * \star P=0.01$; $* * * P<0.001 ;$ n.s. not significant (relative to DMSO controls), one sample $t$-test. Western blots below graphs show examples of MYB protein expression. $(\mathrm{G})$ Western blot analysis of MYB protein expression in AML cells after $24 \mathrm{~h}$ treatment with DMSO, ARQ531 (0.3-1 $\mu \mathrm{M})$ or ARQ531 and $10 \mu \mathrm{M}$ MG132, a proteasome inhibitor.

changes in AML-treated cells. As shown in Figure 4C, ARO531 exposure resulted in a prompt decrease of phosphorylation, followed by a reduction of MYC protein. Accordingly, numerous MYC-addicted oncogenic cellular pathways, such as protein folding machinery, metabolic dependency and genome integrity, were compromised following this treatment, as highlighted by phosphoeukaryotic translation initiation factor 4E (eIF4e), ASCT2 and GLUT1 downregulation and $\gamma \mathrm{H} 2 \mathrm{AX}$ enhancement, respectively (Figure 4D). Combined drug screening revealed synergistic activity of ARO531 with compounds affecting these programs, such as DNA damaging agents (Online Supplementary Figure S6A, B). Overall, these data support the existence of a mechanism of action that begins with MAPK signaling dysregulation and results in ARO531-induced cytotoxicity in AML cells. Among MYC-controlled programs, protein synthesis is emerging as the limiting step for tumor cell growth, ${ }^{36}$ so we focused on this pathway. As shown in Figure 4D, AML cells treat- ed with ARQ531 showed marked increases of eukaryotic translation initiation factor 4E-binding protein (4EBP1) with concomitant de-phosphorylation of p70 ribosomal S6 kinase (p70-S6K) and eIF4e which result in blocking of mRNA recruitment to ribosomes for protein translation. ${ }^{37}$ These data suggest that ARO531 is a modulator of several hubs controlling translation initiation in AML cells, providing evidence of marked protein synthesis inhibition specifically triggered by this treatment.

Although MYC activation resulting from multiple tumor-driven genetic aberrations has been recognized as a major factor of leukemogenesis, its targeting did not show significant clinical benefit in AML. Thus, by using a small interference RNA (siRNA) strategy, we investigated the role of MYC in the anti-leukemic activity of ARQ531. As shown in Figure 4E, MYC-silenced HL-60 cells (MYC knocked down) were treated with increasing doses of ARO531. Surprisingly, despite their sensitivity to this treatment, the cells were quite resistant to the loss of 
A

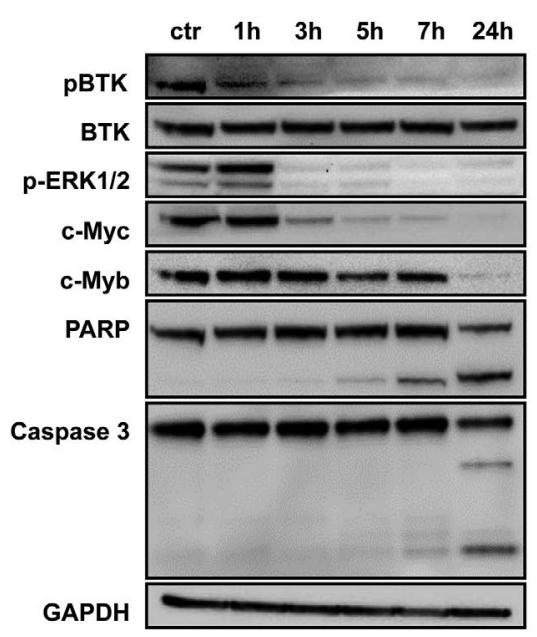

B

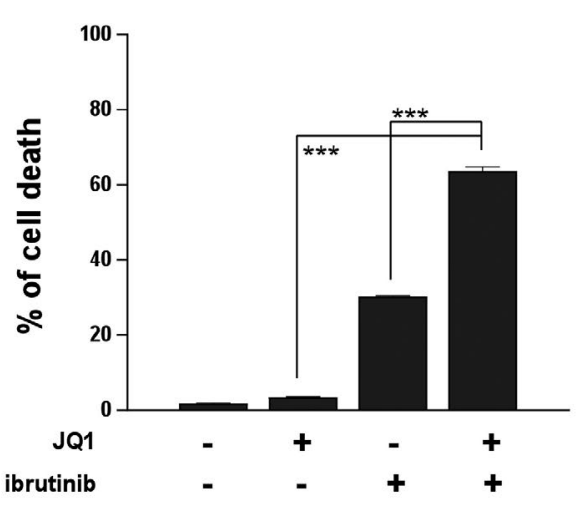

C

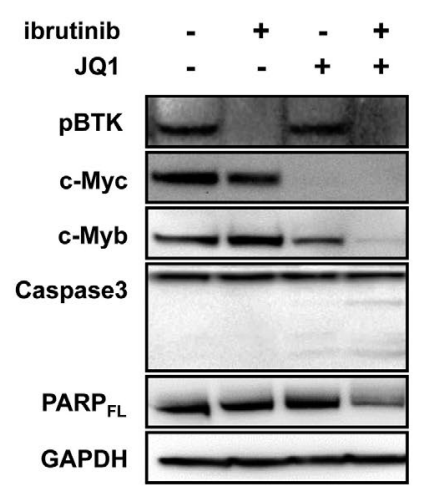

Figure 5. ARQ531 treatment results in oncogenic program dysregulation in acute myeloid leukemia cells. (A) Western blot analysis of cells treated with ARQ531 (1 $\mu \mathrm{M})$ after the indicated hours. Time-dependent effects demonstrate early inhibition of BTK activity and MYC downregulation followed by a reduction of MYB with associated PARP and caspase 3 cleavage. (B) Treatment of HL60 cells with BET bromodomain inhibitor JQ1 ( $400 \mathrm{nM}$ ), ibrutinib (30 $\mu \mathrm{M})$ or their combination, which resulted in a synergistic effect. Bars and error bars are means and standard deviation of three independent experiments. $* * * P<0.001$ (relative to dimethylsulfoxide controls), one-sample $t$-test. (C) Western blot showing that ibrutinib, JQ1, and their combination result in appearance of apoptotic features, including caspase 3 and PARP cleavage in HL60 cells.

MYC protein expression, indicating that additional targets are implicated in anti-AML activity of this small molecule.

Modulation of transcriptional regulatory machinery is an innovative strategy to treat AML. ${ }^{38,39}$ The oncogenic driver MYB, which is essential in hematopoiesis, is now emerging as a new target for anti-AML therapies. $40.45 \mathrm{We}$ hypothesized that ARQ531 treatment of AML cells may inhibit this pathway. To validate this hypothesis, we measured MYB protein levels in ARO531-treated cells. Exposure to ARO531 resulted in marked MYB deregulation (Figure 4F), suggesting an important contribution to ARO531 anti-tumor activity. To gain further insights into MYB reduction triggered by ARQ531, we tested the proteasome contribution, as previously reported for other MYB-targeting agents. ${ }^{43}$ As shown in Figure 4G, co-treatment with the proteasome inhibitor MG132 preserved MYB protein levels, suggesting that, in addition to its supposed effects on protein synthesis, ARO531 affects MYB degradation. Our findings therefore suggest that ARO531 interferes with many pro-survival pathways, such as MAPK, in AML cells.

\section{ARQ531 dysregulates multiple oncogenic transcription} factors in acute myeloid leukemia cells

To gain insights into the molecular mechanisms of ARO531, we analyzed treated HL-60 cells over time. As shown in Figure 5A, BTK signaling deregulation occurred early, after $2 \mathrm{~h}$ of treatment, followed by MYC downregulation. Importantly, apoptotic cell features, including PARP and caspase 3 cleavage, were seen after the decrease in MYB, suggesting that these events are crucial for ARO531 anti-tumor activity. Published data show that small molecule BET inhibitors, by downregulating hematopoietic transcription factors, lead to potent therapeutic effects in several cancer models, including AML ${ }^{46,47}$ We therefore tested the anti-AML activity of the BTK inhibitor ibrutinib combined with the BET bromodomain inhibitor JO1. ${ }^{48}$ As was seen in other cell types, ${ }^{25}$ the BET inhibitor enhanced the anti-tumor activity of the BTK inhibitor (Figure 5B). Western blot analysis of AML-treated cells confirmed these findings, further supporting the pivotal role of transcription factor deregulation in the anti-AML activity of ARO531 (Figure 5C). Based on these data, we investigated the role of MYB in ARO531 antiAML activity by challenging BTK-silenced cells with the repurposed drug mebendazole, recently described as a drug that induces MYB degradation..$^{44}$ As expected, mebendazole reduced cell viability of BTK-depleted cells more than a control (Online Supplementary Figure S7A). We then performed several genetic studies to confirm these findings. As shown in Figure 6A, reduced viability was observed in MYC/MYB-depleted cells compared with the control, but more importantly, viability was significantly dampened in triple MYB/MYC/BTK-silenced cells (reduction by $64.7 \%$ to $38.5 \%$ ), suggesting that such inhibition is detrimental to AML cells. Consistently, simultaneous silencing of MYC, MYB and BTK resulted in PARP cleavage together with impairment of ERK phosphorylation (Figure 6B). Similar data were observed in BTK-KD cells (Figure 6C and Online Supplementary Figure S7B). Nonetheless, the effect of triple knockdown was not quite equal to that of ARO531 treatment, suggesting that other covalent or noncovalent targets are involved in the drug's mechanism of action.

Since MYB is reported to be crucial for leukemogenesis $^{42,49}$ we assessed the relationship between BTK and MYB in AML cells. Molecular data analysis of different publicly available AML cohort databases revealed higher expression of BTK and MYB in AML cells compared to normal HSC, with a positive correlation (Online Supplementary Figure S8A-C). These data support the notion that several oncogenic pathways, including BTK, MYB and MYC, are essential for leukemia cell maintenance, supporting the concept that ARQ531 could be an effective multi-targeted agent for the treatment of AML. 
A

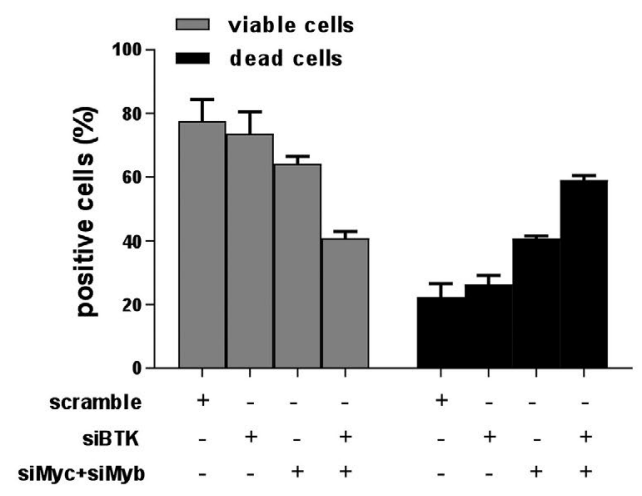

B

C
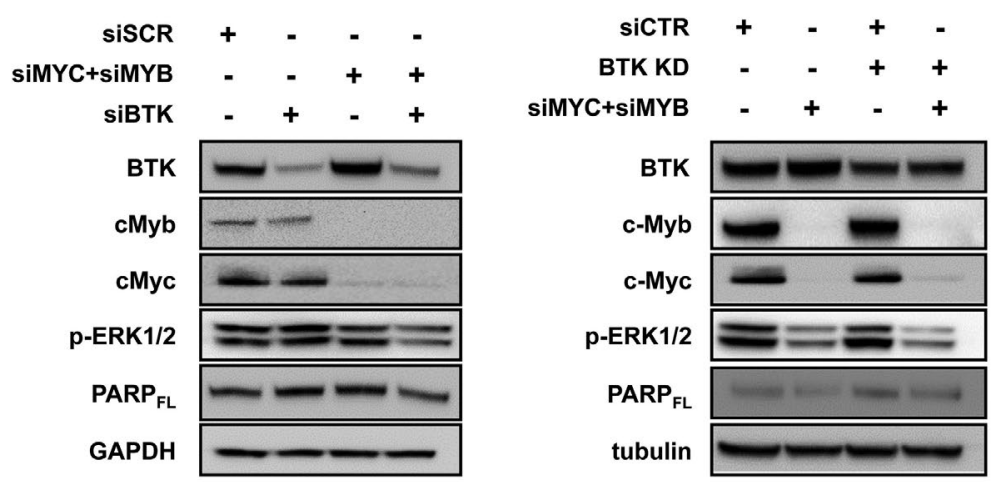

Figure 6. ARQ531 affects BTK, MYC and MYB in acute myeloid leukemia cells. (A) BTK, MYC, MYB, and especially their simultaneous silencing considerably reduced viability of HL60 cells as measured by Trypan blue staining. The mean of triplicate experiments are shown. (B, C) Triple BTK/MYC/MYB-silenced HL60 cells demonstrate diminished phosphorylation of ERK and PARP full-length form compared with cells depleted either transiently (B) or stably (C) of each gene, individually.

\section{ARQ531 shows potent activity in a patient-derived xenograft mouse model of acute myeloid leukemia}

Based on in vitro data, we next assessed whether ARO531 treatment would be effective and tolerable in animal models by using our established AML patient-derived xenograft model. NSG mice $(n=20)$ were engrafted with 300,000 primary human AML cells (M4, acute myelomonocytic leukemia). Successful engraftment was documented by measuring circulating human $\mathrm{CD}_{4} 5^{+}\left(\mathrm{hCD} 45^{+}\right)$cells in the animals' peripheral blood with flow cytometry weekly for 2 months. At day 19 after infusion of the cells, once a systemic xenograft had been confirmed, mice were dosed orally with vehicle or ARO531 ( 25 or $37.5 \mathrm{mg} / \mathrm{kg}$; 5 mice/group) daily for 2 weeks. The percentage of human cells in peripheral blood samples was measured once a week up to day 42 (Figure 7A). ARO531-treated mice had significant reductions in the numbers of $\mathrm{hCD} 45^{+}$cells despite very rapid growth of the aggressive leukemic cells (Figure 7B). At day 42 after starting treatment, there were $66.5 \pm 0.1 \%$ and $69.5 \pm 0.2 \%$ hCD45+ cells after ARO531 treatment at the doses of 37.5 and $25 \mathrm{mg} / \mathrm{kg}$, respectively; in contrast, vehicle-treated mice had $85 \%$ hCD $45^{+}$cells $\left({ }^{* *} 0.005<P<0.008\right)$ (Figure $7 \mathrm{C}$ ). Analyses of bone marrow and spleen also showed reductions in tumor burden $\left(\mathrm{hCD} 45^{+}\right)$, although they were not statistically significant (Online Supplementary Figure S9). In addition, ARQ531 treatment was found to improve mouse survival significantly. As shown in Figure 7D, Kaplan-Meier analyses indicated that mice treated with the higher dose of ARO531 survived significantly longer than those treated with the vehicle control $(P<0.001)$. Overall, treatment was well tolerated as suggested by the maintenance of body weight and the lack of signs of toxicity, such as lethargy, ruffled fur, respiratory distress and hunchback posture (data not shown). Together these data indicate that, in vivo, ARO531 administration was well tolerated and efficiently reduced leukemia cell growth, providing impetus for clinical evaluation of this novel small molecule.

\section{Discussion}

AML cells often demonstrate constitutive activation of tyrosine kinase signaling resulting from specific genomic aberrations ${ }^{16}$ These aberrations are attractive therapeutic targets, as demonstrated by the pharmacological inhibitor of BTK, ibrutinib, which blocks AML blast proliferation, migration, and leukemic cell adhesion to bone marrow stromal cells..$^{15}$ However, BTK-based treatment of AML patients has been unsuccessful to date, ${ }^{22}$ with only a few preclinical, ex vivo studies suggesting that ibrutinib is effective against FLT3(ITD) and CD117 harboring cells, unlike the clinical benefit seen in patients with chronic lymphocytic leukemia and lymphoma. ${ }^{18,21}$ Adding inhibitory pressure on the BTK pathway might enhance the efficacy of this strategy, as previously reported. . $920,50.54^{2}$ In this study, using a combination of genetic and biochemical approaches, we extensively characterized ARO531, a novel, reversible, orally bioavailable, ATP-competitive inhibitor of BTK and associated kinases. ARO531 greatly compromises AML cell survival by modulating transcriptional regulatory machinery coordinated by MYC, demonstrating activity both in vitro and in a patient-derived xenograft AML mouse model. Thus, our study provides the rationale for developing clinical trials using ARQ531 as a new treatment for patients with AML.

Since ibrutinib does not directly inhibit components of the MAPK pathway, it is possible that the superior activity of ARQ531 in AML may be due to its modulation of additional targets, including kinases related to ERK signaling. ${ }^{27}$ Although screening analysis of Src-family kinases (including Lyn and Syk) did not show any effect on AML cells, ${ }^{55}$. ${ }^{57}$ (Online Supplementary Figure S10) we assume that targeting of additional kinases is responsible for the marked anti-AML activity of ARO531. By combining computational models and whole transcriptional analysis, we observed that ARO531 treatment induces dysregulation of several transcription-addicted programs, including MYC and MYB. The combination of BTK inhibition and MYC/MYB downregulation explains the improved antiAML activity of ARQ531 compared to single agent tyrosine kinase inhibitors such as ibrutinib. Since ARO531 simultaneously inhibits different cellular functions such as folding machinery, metabolic dependency, and genome integrity, it may provide deeper and more durable remissions, while delaying the emergence of resistance. Additionally, based on reports that degrading MYB eradi- 
A

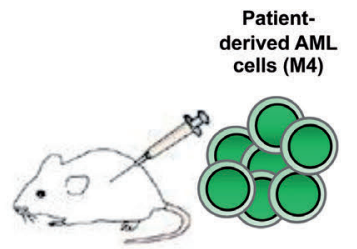

LEUKEMIA PROGRESSION
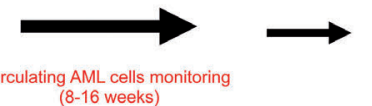

ARQ531

$(25 \mathrm{mg} / \mathrm{kg})$

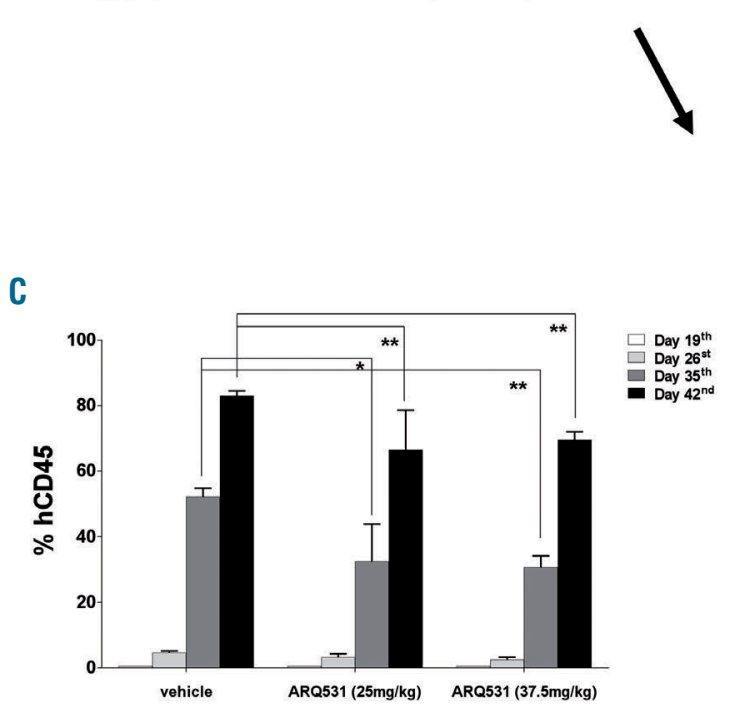

ARQ531

(37.5 mg/kg)

C

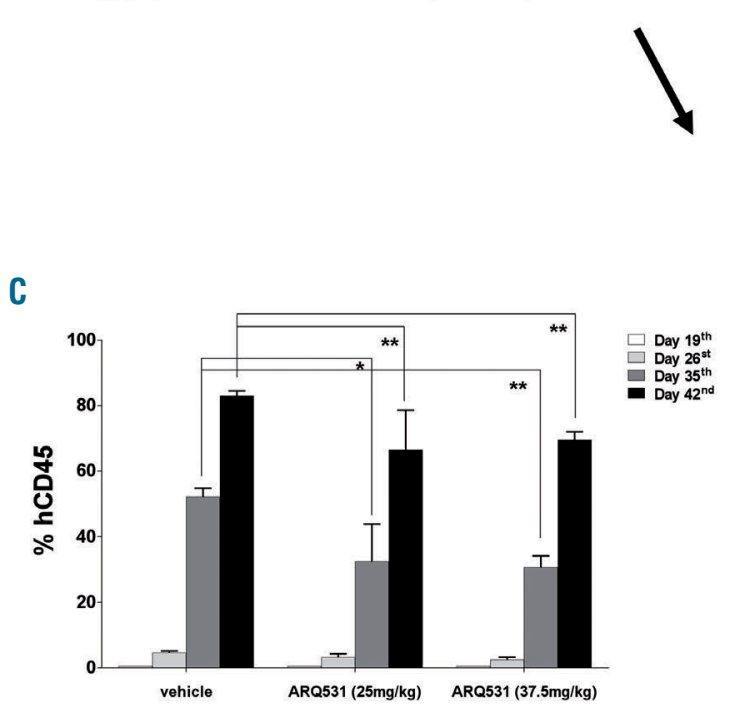

TREATMENT (2 weeks)

D

D
B
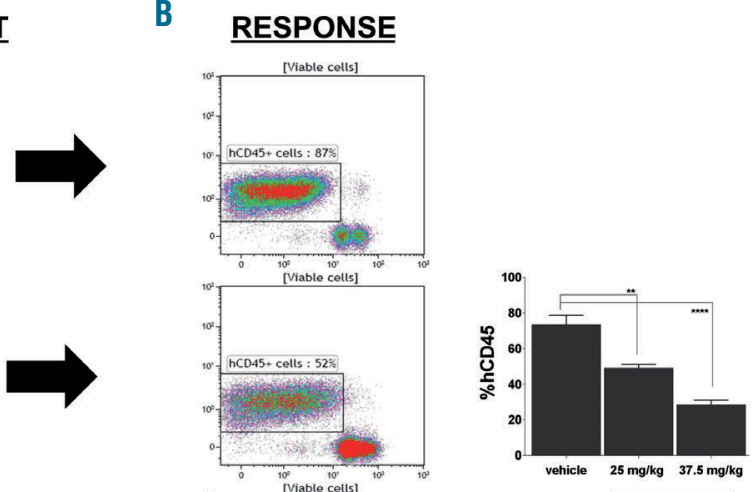

ARQ531
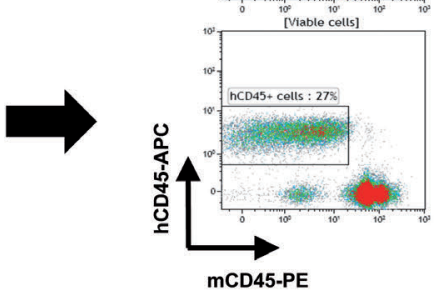

mCD45-PE

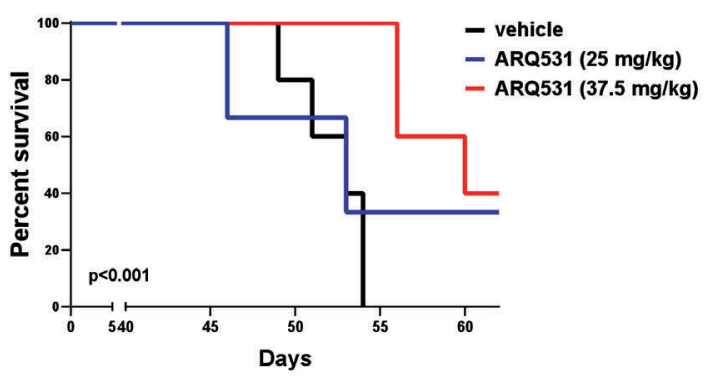

Figure 7. ARQ531 inhibits tumor growth and extends survival in a patient-derived xenograft mouse model of acute myeloid leukemia. (A) Experimental outline for the analysis of the anti-leukemic activity of ARQ531 against primary human acute myeloid leukemia (AML) cells. A patient-derived xenograft mouse model of human primary AML cells was used to assess the efficacy of ARQ531 against AML cells isolated from patients with AML M4. (B) Representative flow cytometric dot plots representing tumor engraftment evaluated at day 35 after treatment. In the right panel, the histogram represents the percentage of human $\mathrm{CD} 45^{+}$cells in mice. Data are represented as mean \pm standard deviation; $* * P=0.006 ; * * * * P<0.001$. (C) Circulating human CD45 cells were measured in peripheral blood by flow cytometry weekly for 2 months. At day 19, a systemic xenograft was confirmed (tumor engraftment) and mice were randomized to receive vehicle control, a low dose of AR0531 (25 mg/kg) or a high dose of ARQ531 (37.5 mg/kg). The percentage of human leukemic cells in peripheral blood of mice was measured weekly, up to day 42 . $0.005<* * P<0.008$. (D) Kaplan-Meier curve of the patient-derived xenograft AML model following treatment with vehicle, a low dose of ARQ531 (25 mg/kg) or a high dose of ARQ531 (37.5 mg/kg). The higher drug dose led to significantly longer overall survival compared to that of the vehicle-treated, control mice (5 mice/group; $P<0.001)$.

cates AML cells in mice without impairing normal myelopoiesis, ${ }^{46}$ ARQ531 treatment may be safe for hematopoietic precursor cells, supporting its clinical relevance. We also provide experimental evidence that the bone marrow stroma is not affected by treatment and, more importantly, does not affect the anti-tumor activity of ARQ531. Preliminary phase I studies confirm the safety profile of ARO531, adding to the data that support its clinical development.

Recent studies suggest that modulating transcriptional regulatory machinery is an innovative strategy to treat blood malignancies, including AML. ${ }^{14,38}$ An example of this strategy is all-trans retinoic acid treatment which, by modulating the transcriptional target PML-RAR $\alpha$, induces differentiation of leukemic blasts resulting in improved survival of patients. ${ }^{58}$ However, most transcription factors remain notoriously difficult to target, with siRNA-mediated silencing of gene expression being one of the few feasible approaches. ${ }^{59}$ Other oncoproteins, including MYC and $M Y B$, are emerging as compelling targets for drug development in AML, due to their ability to influence tumor proliferation. ${ }^{40-44,60,61}$ In this context, the new small molecule ARQ531, by affecting multiple oncogenic pathways simultaneously, results in perturbation of the transcriptional regulatory machinery which maintains AML cell integrity. Therefore, targeting BTK, MYC and MYB with ARO531 represents an innovative strategy for improving the efficacy of AML therapy.

In summary, we have demonstrated that ARO531, a new reversible tyrosine kinase inhibitor, suppresses AML cell viability in vitro and in vivo by abrogating different oncogenic targets including BTK, MYC and MYB. Gene silencing of BTK, MYC and MYB in AML cells was not as effective as ARO531, suggesting that other covalent or noncovalent targets are involved in its mechanism of action. Based on our preclinical data, we provide the rationale to explore the effects of this multi-targeted agent on hematologic malignancies as well as solid tumors, beyond investigating its clinical benefit in AML patients. 


\section{Acknowledgments}

This work was supported in part by the Associazione Italiana per la Ricerca sul Cancro (AIRC, MYFG \#18491 to $M C$ and \#21552 to AC; I.G. to FB), Italian Ministry of
Health $(5 \times 1000$ Funds of IRCCS San Martino-IST 2014 and 2015, to MC and AC), Associazione Italiana Leucemie Linfomi e Mieloma (AIL sezione di Genova) and University of Genoa, Italy.

\section{References}

1. Sant M, Allemani C, Tereanu C, et al. Incidence of hematologic malignancies in Europe by morphologic subtype: results of the HAEMACARE project. Blood. 2010;116 (19):3724-3734

2. Ferrara F, Schiffer CA. Acute myeloid leukaemia in adults. Lancet. 2013;381 (9865):484-495.

3. Esposito MT, So CW. DNA damage accumulation and repair defects in acute myeloid leukemia: implications for pathogenesis, disease progression, and chemotherapy resistance. Chromosoma. 2014;123(6):545-561.

4. Stein EM. FLT3 inhibitors for relapsed or refractory acute myeloid leukaemia. Lancet Oncol. 2018;19(7):849-850

5. El Fakih R, Rasheed W, Hawsawi Y, Alsermani M, Hassanein M. Targeting FLT3 mutations in acute myeloid leukemia. Cells. 2018;7(1):4

6. DiNardo CD, Stein EM, de Botton S, et al. Durable remissions with ivosidenib in IDH1-mutated relapsed or refractory AML. N Engl J Med. 2018;378(25):2386-2398.

7. Buggy JJ, Elias L. Bruton tyrosine kinase (BTK) and its role in B-cell malignancy. Int Rev Immunol. 2012;31(2):119-132.

8. Hendriks RW, Bredius RG, Pike-Overzet K, Staal FJ. Biology and novel treatment options for XLA, the most common monogenetic immunodeficiency in man. Expert Opin Ther Targets. 2011;15(8):1003-1021.

9. Herman SEM, Montraveta A, Niemann CU, et al. The Bruton tyrosine kinase (BTK) inhibitor acalabrutinib demonstrates potent on-target effects and efficacy in two mouse models of chronic lymphocytic leukemia. Clin Cancer Res. 2017;23(11):2831-2341.

10. Wilson WH, Young RM, Schmitz R, et al. Targeting B cell receptor signaling with ibrutinib in diffuse large B cell lymphoma. Nat Med. 2015;21(8):922-926.

11. Advani RH, Buggy JJ, Sharman JP, et al. Bruton tyrosine kinase inhibitor ibrutinib (PCI-32765) has significant activity in patients with relapsed/refractory B-cell malignancies. J Clin Oncol. 2013;31(1):8894.

12. Treon SP, Tripsas CK, Meid K, et al. Ibrutinib in previously treated Waldenstrom's macroglobulinemia. N Engl J Med. 2015;372(15):1430-1340.

13. Rushworth SA, Bowles KM, Barrera LN, Murray MY, Zaitseva L, MacEwan DJ. BTK inhibitor ibrutinib is cytotoxic to myeloma and potently enhances bortezomib and lenalidomide activities through NF-kappaB. Cell Signal. 2013;25(1):106-112.

14. Grommes C, Pastore A, Palaskas N, et al. Ibrutinib unmasks critical role of Bruton tyrosine kinase in primary CNS lymphoma. Cancer Discov. 2017;7(9): 10181029.

15. Rushworth SA, Murray MY, Zaitseva L, Bowles KM, MacEwan DJ. Identification of Bruton's tyrosine kinase as a therapeutic target in acute myeloid leukemia. Blood. 2014;123(8):1229-1238.

16. Oellerich T, Mohr S, Corso J, et al. FLT3-
ITD and TLR9 use Bruton tyrosine kinase to activate distinct transcriptional programs mediating AML cell survival and proliferation. Blood. 2015;125(12):1936-1947

17. Wu H, Hu C, Wang A, et al. Ibrutinib selectively targets FLT3-ITD in mutant FLT3positive AML. Leukemia. 2016;30(3):754 757.

18. Rushworth SA, Pillinger G, Abdul-Aziz A et al. Activity of Bruton's tyrosine-kinase inhibitor ibrutinib in patients with CD117. positive acute myeloid leukaemia: a mechanistic study using patient-derived blast cells. Lancet Haematol. 2015;2(5):e204-211.

19. Rotin LE, Gronda M, MacLean N, et al. Ibrutinib synergizes with poly(ADP-ribose) glycohydrolase inhibitors to induce cell death in AML cells via a BTK-independent mechanism. Oncotarget. 2016;7(3):27652779

20. Li X, Yin X, Wang $\mathrm{H}$, et al. The combination effect of homoharringtonine and ibrutinib on FLT3-ITD mutant acute myeloid leukemia. Oncotarget. 2017;8(8):1276412774

21. Pillinger G, Abdul-Aziz A, Zaitseva L, et al Targeting BTK for the treatment of FLT3 ITD mutated acute myeloid leukemia. Sci Rep. 2015;5:12949.

22. Cortes JE, Estey E, Stein AS, Graef T, et al. A multicenter, open-label phase 2 a study of ibrutinib with or without cytarabine in patients with acute myeloid leukemia (PCYC-1131). J Clin Oncol. 2015;33 (15_suppl):TPS7096-TPS7096.

23. Chong IY, Aronson L, Bryant H, et al Mapping genetic vulnerabilities reveals BTK as a novel therapeutic target in oesophageal cancer. Gut. 2018;67(10):17801792

24. Moyo TK, Wilson CS, Moore DJ, Eischen CM. Myc enhances B-cell receptor signaling in precancerous $B$ cells and confers resistance to Btk inhibition. Oncogene. 2017;36(32):4653-4661

25. Sun B, Shah B, Fiskus W, Oi J, Rajapakshe $\mathrm{K}$, Coarfa C, et al. Synergistic activity of BET protein antagonist-based combinations in mantle cell lymphoma cells sensitive or resistant to ibrutinib. Blood. 2015;126(13):1565-1574.

26. Sasi BK, Martines C, Xerxa E, et al Inhibition of SYK or BTK augments venetoclax sensitivity in SHP1-negative/BCL-2 positive diffuse large B-cell lymphoma. Leukemia. 2019;33(10):2416-2428.

27. Reiff SD, Mantel R, Smith LL, et al. The BTK inhibitor ARO 531 targets ibrutinibresistant CLL and Richter transformation. Cancer Discov. 2018;8(10):1300-1315.

28. Lane SW, Scadden DT, Gilliland DG. The leukemic stem cell niche: current concepts and therapeutic opportunities. Blood. 2009;114(6):1150-1157.

29. Bam R, Ling W, Khan S, et al. Role of Bruton's tyrosine kinase in myeloma cell migration and induction of bone disease. Am J Hematol. 2013;88(6):463-471.

30. Chang BY, Francesco M, De Rooij MF, et al Egress of CD19(+)CD5(+) cells into peripheral blood following treatment with the Bruton tyrosine kinase inhibitor ibrutinib in mantle cell lymphoma patients. Blood. 2013;122(14):2412-2424.

31. Zaitseva L, Murray MY, Shafat MS, et al. Ibrutinib inhibits SDF1/CXCR4 mediated migration in AML. Oncotarget. 2014;5(20): 9930-9938.

32. Dhillon AS, Hagan S, Rath O, Kolch W. MAP kinase signalling pathways in cancer Oncogene. 2007;26(22):3279-3290.

33. Lamba S, Russo M, Sun C, et al. RAF suppression synergizes with MEK inhibition in KRAS mutant cancer cells. Cell Rep. 2014;8(5):1475-1483.

34. McCubrey JA, Steelman LS, Franklin RA, et al. Targeting the RAF/MEK/ERK, PI3K/AKT and p53 pathways in hematopoietic drug resistance. Adv Enzyme Regul. 2007:47:64-103.

35. Sears R, Nuckolls F, Haura E, Taya Y, Tamai K, Nevins JR. Multiple Ras-dependent phosphorylation pathways regulate Myc protein stability. Genes Dev. 2000;14(19): 2501-2514.

36. Sonenberg N, Hinnebusch AG. Regulation of translation initiation in eukaryotes: mechanisms and biological targets. Cell. 2009:136(4):731-745.

37.Zucal C, D'Agostino VG, Casini A, et al. EIF2A-dependent translational arrest protects leukemia cells from the energetic stress induced by NAMPT inhibition. BMC Cancer. 2015;15:855.

38. Brien GL, Valerio DG, Armstrong SA Exploiting the epigenome to control cancerpromoting gene-expression programs. Cancer Cell. 2016;29(4):464-476.

39. Li S, Vallet S, Sacco A, Roccaro A, Lentzsch S, Podar K. Targeting transcription factors in multiple myeloma: evolving therapeutic strategies. Expert Opin Investig Drugs. 2019;28(5):445-462.

40. Coulibaly A, Haas A, Steinmann S, Jakobs A, Schmidt TJ, Klempnauer KH. The natural anti-tumor compound Celastrol targets a Myb-C/EBPbeta-p300 transcriptional module implicated in myeloid gene expression. PLoS One. 2018;13(2):e0190934.

41. Ramaswamy K, Forbes L, Minuesa G, et al. Peptidomimetic blockade of MYB in acute myeloid leukemia. Nat Commun. 2018;9 (1):110.

42. Xu Y, Milazzo JP, Somerville TDD, et al. A TFIID-SAGA Perturbation that targets MYB and suppresses acute myeloid leukemia. Cancer Cell. 2018;33(1):13-28 e8.

43. Walf-Vorderwulbecke V, Pearce K, Brooks $\mathrm{T}$, et al. Targeting acute myeloid leukemia by drug-induced c-MYB degradation. Leukemia. 2018;32(4):882-889.

44. Liu W, Wu M, Huang Z, et al. c-myb hyperactivity leads to myeloid and lymphoid malignancies in zebrafish. Leukemia. 2017;31(1):222-233

45. Uttarkar S, Dasse E, Coulibaly A, et al. Targeting acute myeloid leukemia with a small molecule inhibitor of the Myb/p300 interaction. Blood. 2016;127(9):1173-1182.

46. Roe IS, Mercan F, Rivera K, Pappin DJ, Vakoc CR. BET bromodomain inhibition suppresses the function of hematopoietic transcription factors in acute myeloid 
Preclinical activity of ARQ531 in AML

leukemia. Mol Cell. 2015;58(6):1028-1039.

47. Zuber J, She J, Wang E, et al. RNA screen identifies Brd4 as a therapeutic target in acute myeloid leukaemia. Nature. 2011;478 (7370):524-528.

48. Filippakopoulos P, Qi J, Picaud S, et al. Selective inhibition of BET bromodomains. Nature. 2010;468(7327):1067-1073.

49. Pattabiraman DR, Gouda TJ. Role and potential for therapeutic targeting of MYB in leukemia. Leukemia. 2013;27(2):269-277.

50. Linley A, Krysov S, Ponzoni M, Johnson PW, Packham G, Stevenson FK. Lection binding to surface In variable regions provide a universal persistent activating signal for follicular lymphoma cells. Blood. 2015;126(16):1902-1910.

51. Caa M, Cagnetta A, Acharya C, et al. Dual NAMPT and BTK targeting leads to synerfistic killing of Waldenstrom macroglobulinemia cells regardless of MYD88 and CXCR4 somatic mutation status. Olin Cancer Res. 2016;22(24):6099-6109.
52. Deng J, Isik E, Fernandes SM, Brown JR, Letai A, Davids MS. Briton's tyrosine kinase inhibition increases BCL-2 dependence and enhances sensitivity to venetoclax in chronic lymphocytic leukemia. Leukemia. 2017;31(10):2075-2084.

53. Hing ZA, Mantel R, Beckwith KA, et al. Selinexor is effective in acquired resistance to ibrutinib and synergizes with ibrutinib in chronic lymphocytic leukemia. Blood. 2015;125(20):3128-3132.

54. de Rooij MF, Kuil A, Gran W, et al. Ibrutinib and idelalisib target $\mathrm{B}$ cell receptorbut not CXCL12/CXCR4-controlled integrin-mediated adhesion in Waldenstrom macroglobulinemia. Haematological. 2016;101(3):e111-115.

55. Puissant A, Fenouille N, Alexe G, et al. SYK is a critical regulator of FLT3 in acute myeloid leukemia. Cancer Cell. 2014;25(2):226-242.

56. Hahn CK, Berchuck JE, Ross KN, et al. Proteomic and genetic approaches identify
Syk as an AML target. Cancer Cell. 2009;16(4):281-294.

57. Dos Santos C, Demur C, Bardet V, ParadeHoudellier N, Payrastre B, Recher C. A critital role for Lyon in acute myeloid leukemia. Blood. 2008;111(4):2269-2279.

58. Lo-Coco F, Orlando SM, Platzbecker U. Treatment of acute promyelocytic leukemia. N Engl J Med. 2013;369(15):1472.

59. Yarn C, Higgins PJ. Drugging the undruggable: transcription therapy for cancer. Biochim Biophys Acta. 2013;1835(1):7685.

60. Mertz JA, Conery AR, Bryant BM, et al. Targeting MYC dependence in cancer by inhibiting BET bromodomains. Proc Natl Accad Sci U S A. 2011;108(40):16669-16674.

61. Zuber J, Rappaport AR, Lu W, et al. An integrated approach to dissecting oncogene addiction implicates a Myb-coordinated self-renewal program as essential for leukemia maintenance. Genes Div. 2011;25(15):1628-1640.

haematological | 2020; 105(10)

2431 